\title{
Assessment of Remote Laboratory Practices in Engineering Technology Dis- tance Education
}

\section{Dr. Mert Bal, Miami University}

Mert Bal received his PhD degree in Mechanical Engineering from the Eastern Mediterranean University, North Cyprus in 2008. He was a Post-Doctoral Fellow in the University of Western Ontario, and a Visiting Researcher at the National Research Council Canada in London, Ontario, Canada between 2008 and 2010. He was involved in various research projects in the areas of collaborative intelligence, localization and collaborative information processing in wireless sensor networks, intelligent agents, agent-based manufacturing scheduling, systems control and automation, distributed control of holonic systems and integrated manufacturing, agile manufacturing, virtual reality and remote laboratory applications in education. He has authored or co-authored various journal and conference publications in these areas. Mert Bal is currently an Assistant Professor in the Miami University, Department of Engineering Technology, Ohio, United States of America. 


\title{
Assessment of Remote Laboratory Practices in Engineering Technology Distance Education
}

\author{
Mert Bal \\ Miami University \\ Department of Engineering Technology \\ Hamilton, $\mathrm{OH}$
}

\begin{abstract}
This paper presents a preliminary study for assessing the remote laboratory practices for distance-learning in an ETAC/ABET accredited engineering technology program.

This initial study has been conducted using a remote robotics workstation, which is developed by the students and faculty members of the program in order to support hands-on student learning experience in programming full-scale automated industrial robot arms through remote-access from distant geographic locations.

The effectiveness of the developed remote robotics workstation have been assessed through comparing the test scores, laboratory grades and qualitative questionnaire survey results of distance and local students taking the same course. Assessment results are analyzed to determine the role of remote laboratories in achieving the learning outcomes and leveraging students' motivation.
\end{abstract}

\section{Introduction}

In the recent years, distance learning in engineering education has gained popularity as a convenient method to meet the increased demand for engineering science and engineering technology degrees. Many universities and colleges around the world utilize multiple synchronous or asynchronous distance delivery modes for teaching engineering courses to students at various geographic locations.

One of the greatest challenges in distance engineering education is the delivery of the 'hands-on' laboratory practices. The instructional laboratories have always been essential parts of engineering curriculum. Due to the size and the high cost of the laboratory equipment, it is often impractical and unaffordable for universities to provide hands-on laboratory equipment to distance learners. Hence quality delivery of the distance laboratory exercises is a problem demanding solution.

Institutions offering online engineering degrees have implemented various methods to address this problem. Common methods include: providing students Lab Kits that replicate laboratory equipment using inexpensive scale models; utilizing virtual-reality or similar simulation tools to provide a sense of digital lab environment to the distance learners; use of Remote Laboratories that utilize internet to provide distance-learning students real-time remote access for controlling real lab equipment or machines. Most of these methods possess significant advantages in online or distance engineering laboratory instruction. However, each of the method stated above also have significant restrictions. For example: Lab Kits are convenient tools for providing hands-on 
distance education unless the course requires heavy, bulky or complex lab equipment which are impractical to replicate such as: industrial robots; fluid trainers; test instruments; manufacturing machines etc. Virtual Reality or simulation systems are useful to virtually replicate expensive and complex machinery used in distance laboratories, but it lacks the hands-on component in the laboratory experiment, which is highly critical for students' learning ${ }^{1}$.

From these aspects, the Remote Laboratory is a potential technology as it has the ability to fill the gaps in distance laboratory education. Using the internet and World Wide Web, heavy and complex lab equipment could be controlled by students from any location. As it constitutes operating real lab equipment remotely, this approach promises more hands-on feeling to students than simulations. Remote laboratory is a complex system that requires significant development efforts. The effectiveness of this approach is typically a function of the problem domain, curriculum and the technology. In order to determine its effectiveness in distance education, careful assessment studies need to be performed in various engineering disciplines ${ }^{2}$.

Remote laboratories or remote-access labs are often characterized by use of automation and information system technologies to provide remote-access to laboratory instruments and equipment. In terms of the physical laboratory equipment requirements, the remote labs are very similar to hands-on labs. What makes them different from real labs is the distance between the experiment and the experimenter. In real-labs, the equipment might be mediated through computer control, but co-located. By contrast, in remote labs experimenters obtain data by controlling geographically detached equipment. In other words, reality in remote labs is mediated by distance.

Remote laboratories have a great potential to provide affordable real experimental data through sharing experimental devices with a pool of schools ${ }^{3}$. Also, they can extend the capabilities of a conventional laboratory. Along one dimension, its flexibility increases the number of times and places a student can perform experiments ${ }^{4}$.

Over the past decade, there have been significant improvements in the remote lab technologies. Many schools have adopted these technologies as convenient methods to deliver hands-on distance education ${ }^{5}$.

Traditionally students did like many aspects of the interactive aspects of hands-on labs; this suggests there might be a tradeoff between the concreteness of hands-on labs and the convenience of remote labs. Several studies performed for assessing the effectiveness of remote labs in hands-on education have found that there are no significant differences in educational outcomes between remote and hands-on laboratories ${ }^{6,7}$. These studies provide useful information to educators making curriculum decisions. However, the studies have been performed on a small number of students. As a result, they of necessity focused on only some of the variables that might reasonably be expected to affect educational outcomes ${ }^{8}$.

This paper presents a section of current remote laboratory practices implemented in the Miami University focusing on a remote industrial robotics workstation. The workstation has been developed to perform distance laboratory exercises in a senior level distance-learning course on manufacturing systems. The remote robotics workstation is one of the remote laboratory systems designed and developed at Miami University for distance-learning. This paper briefly describes the design and implementation details of this remote robot workstation. A comparative study is presented for assessing the effectiveness of the proposed remote laboratory workstation. The 
performed preliminary study combines basic tools including student laboratory reports, test scores and questionnaire surveys that are designed to record students' response regarding their experience with the remote robotics laboratory station. The assessment results are analyzed using statistical methods and presented in this paper in order to discuss effectiveness of the remote robotics laboratory workstation in distance-learning.

\section{Distance-Learning Laboratory Practices in Miami University}

The Department of Engineering technology at the Miami University of Ohio runs an ETAC/ABET ${ }^{9}$ accredited B.S. Electromechanical Engineering Technology program which has a distance education component that connects with ten community colleges within a 300 mile radius of the host institution. The students from various geographic locations take courses from the main campus remotely via the Interactive Video Distance Learning (IVDL) system.

One of the most challenging aspects of this program is the student laboratory experience. As per, required by the ETAC/ABET, in order for students to fully understand their operation, they need to work on the appropriate equipment for the laboratory component of each course of the program. Laboratory experiments for the program courses are designed for pedagogical purpose in order to meet the educational outcomes discussed by the ABET.

For its distance component, the program uses portable, mobile lab instruments delivered to the distance students for performing the laboratory experiments in the subject areas such as: data acquisition, signal processing, sensors, PLCs, hydraulics and pneumatics, and motor control. Equipment required for these areas are relatively inexpensive and simple so that they can be fabricated and made available for each distance learning student. A summary of these instrumentation-based mobile laboratory practices has been presented in the 2010 ASEE Annual Conference ${ }^{10}$.

Over the past couple of years, the program has also been also utilizing other technologies such as virtual-reality and remote laboratories in delivering laboratory experiences for the subject areas that requires relatively bulky, complex and expensive equipment ${ }^{1}$.

Some example remote laboratory systems used in the program are as follows: Remote bottlefilling station: an automated bottling and packaging line that allows distance learning students control and program automation line from distance; Magnetic Levitation Lab Station: remote control and operation of a PID controlled magnetic levitation equipment; Fluid Trainer Lab Station: a system that allows distance users remotely control and monitor a fluid trainer. Most of the remote laboratory systems used in the program is developed by the program students and faculty members.

The focus of this paper will be given to the Remote Robotics Laboratory Workstation, which is designed for providing remote access to a full-scale industrial robot for remote control and programming purposes. The design and implementation details of this station are explained in the following section. 


\section{Remote Robotics Laboratory Workstation System Overview}

The remote robotic laboratory workstation is an instructional system designed and developed mainly by the students of the program as part of their senior design capstone projects.

The system uses World-Wide-Web to enable distance students access an industrial robot arm manipulator located in one of the laboratories on campus. The robot arm manipulator used for this project is a five-axis Mitsubishi Movemaster RV-M1 robot with $1.2 \mathrm{~kg}$ lifting capacity ${ }^{11}$. The control device of the robot is equipped with the card containing 16 binary inputs and outputs. It also connects to a personal computer, using Centronics or RS232 port. Despite its small size, the robot constitutes a typical industrial machine. The robot is normally programmed using a personal computer and a teach pendant. The teach pendant is used for manually controlling the robot and teaching positions.

The system uses a web-controllable AXIS ${ }^{12}$ network camera providing a visual feedback to the students while they control the robot arm. The web-camera utilizes a separate IP address for connecting to web server, and provides its client users a remote, real-time access for the captured images. The camera is also equipped with a pan \& tilt system. Students can remotely move the camera, pan, tilt and adjust their view angles as needed. Figure 1 shows the hardware layout of the system.

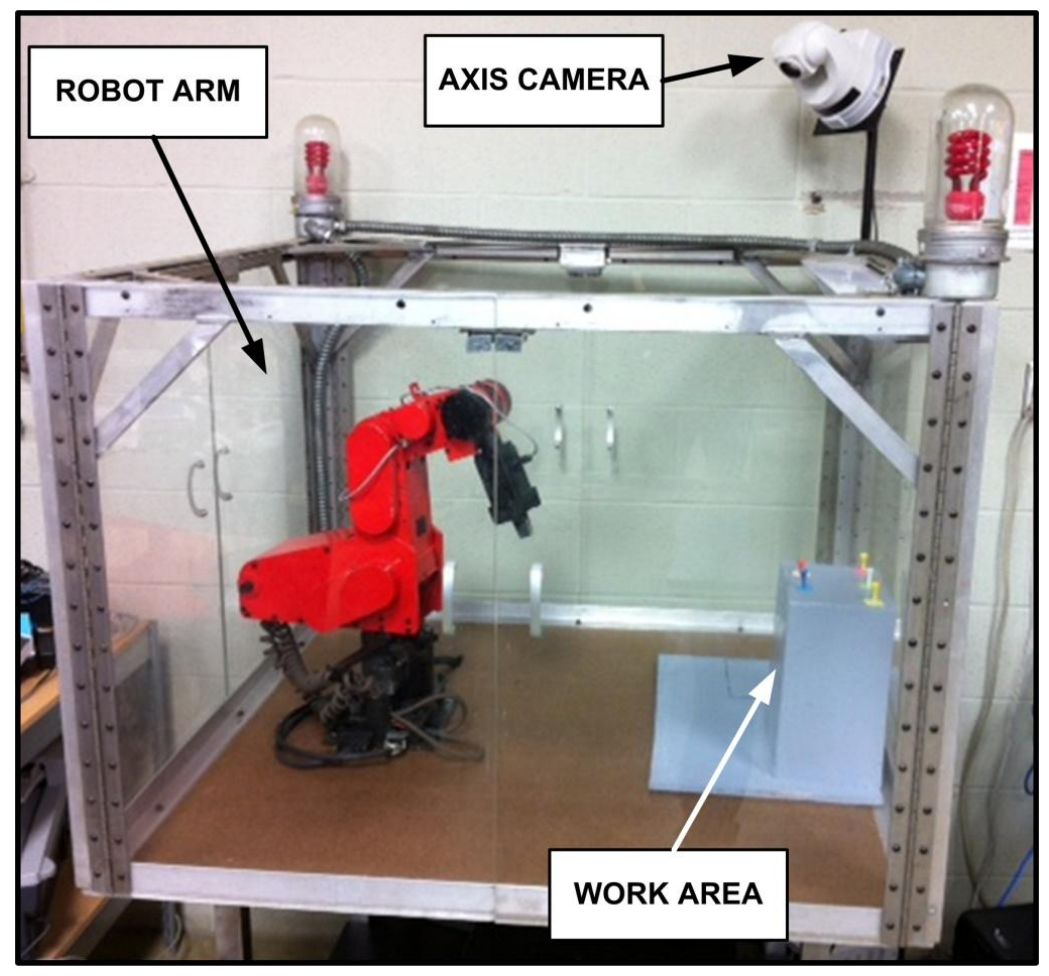

Figure 1: Remote Robotics System Hardware

The hardware architecture for the presented remote laboratory system is shown in the Figure 2. The system is based on a client-server network approach that allows the concurrent execution of multiple experiments using separate experimental setups. Multiple experiments requiring the 
same experimental setup are queued and executed in batch mode in the order of the incoming requests.

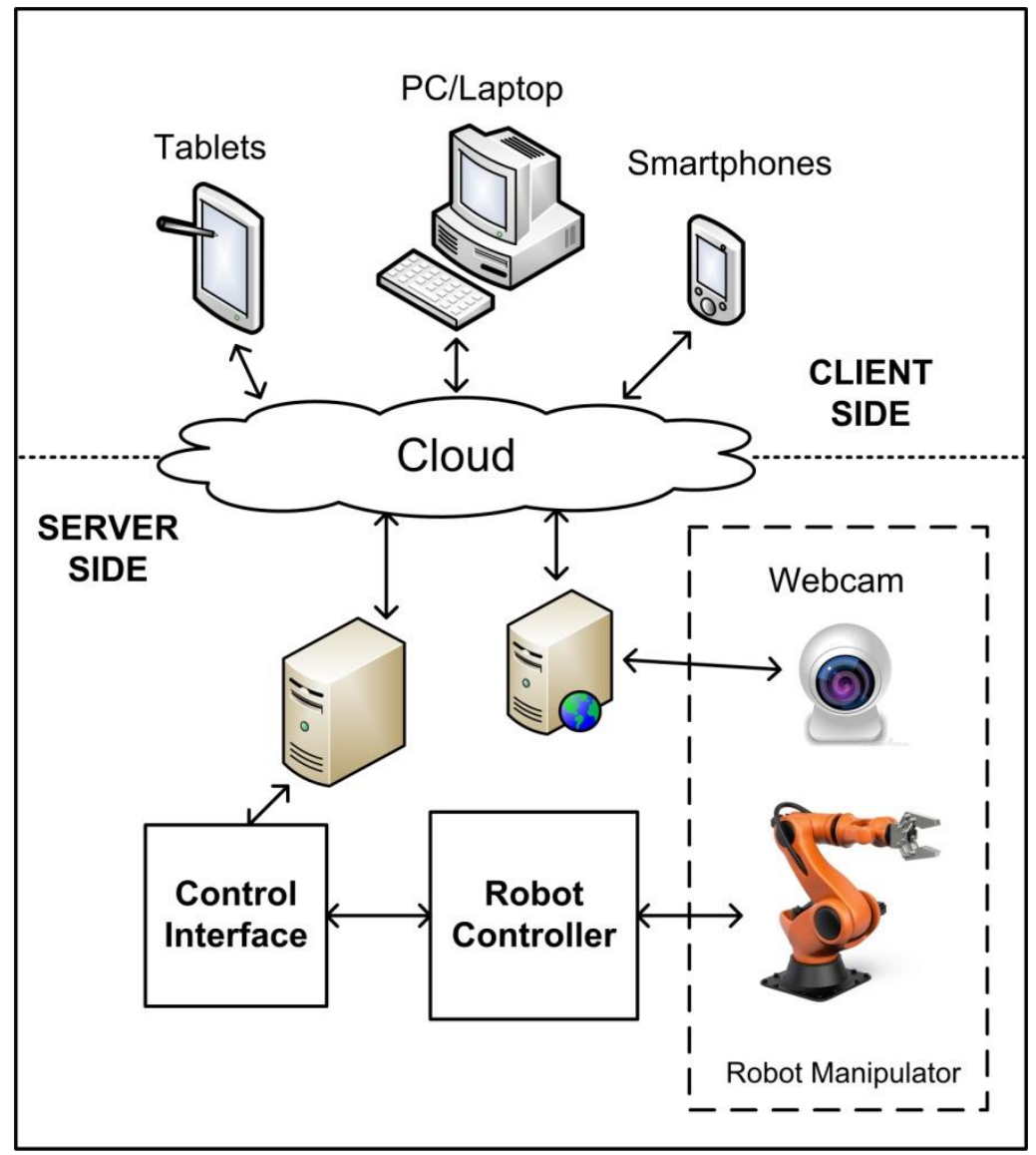

Figure 2: Network System Architecture of the Presented Remote Robotics System

The Server computer running Microsoft Windows 7 operating system uses a Static Internet Protocol (Static IP) address for client-server communication. A server-side application was developed in Visual Basic.NET language and deployed to the server computer to run as a listener for requests from the clients. When a communication request is sent from a client application, a port number will be assigned to the socket program through which the communication between the server and client application can be established.

The server-side application serves as a bridge between the client-side application and the robot controller. After certain formatting is done, the server forwards clients requests to the robot controller and bridges robot controller status back to the clients.

The client-side application is mainly used for communicating the robot controller directly through a control panel to allow students control the robot arm manipulator.

Student users will have to know the right credentials to login into the client application before the robot control panel will be available to them. The robot control panel on the client-side application was designed to provide easy-access to the students in controlling the robot axes (See Figure 3). 
On this interface, students can manually move the robot arm manipulator various positions on its work area and record robot positions. They can also use a programming interface to enter robot programs by typing them on the screen. Once the students complete a robot program, they can send it to the robot through the web server and test the program by observing the robot in action through the web camera (See Figure 4).

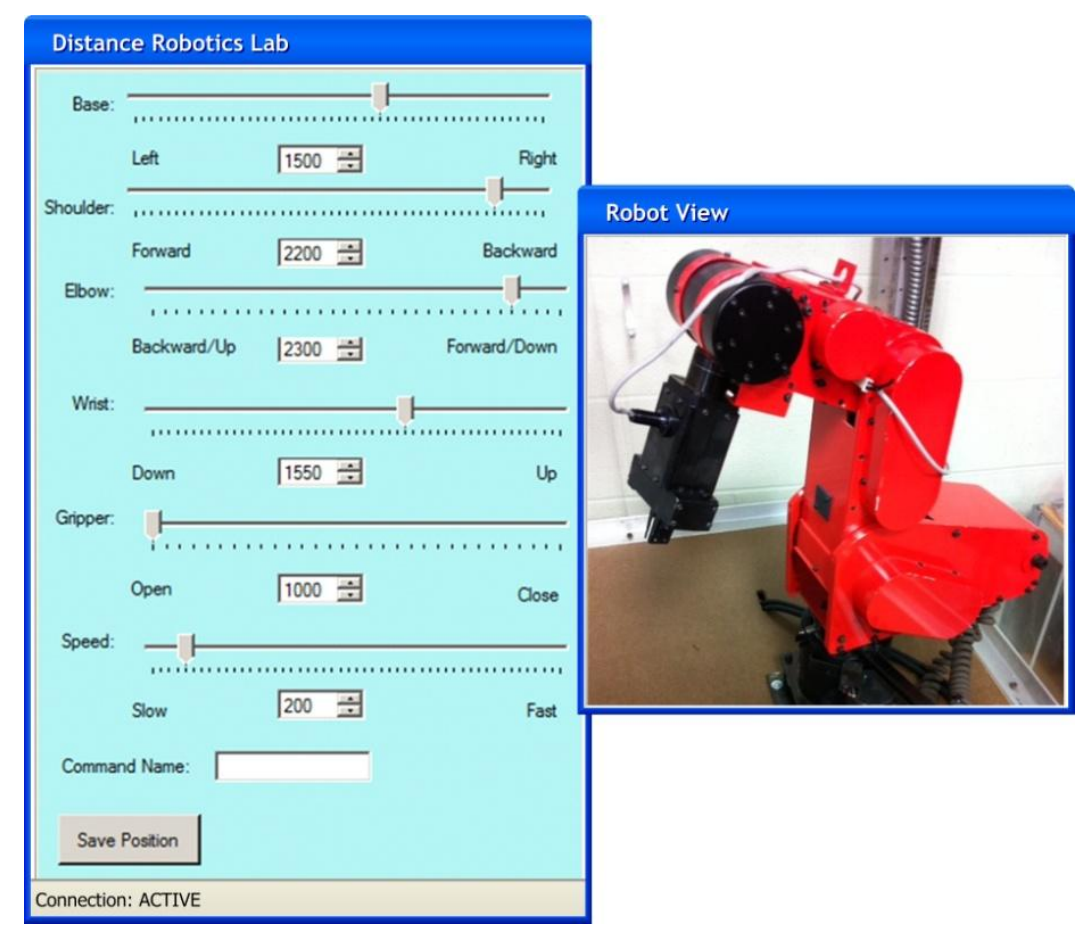

Figure 3: Client-side application interface in CONTROL mode 


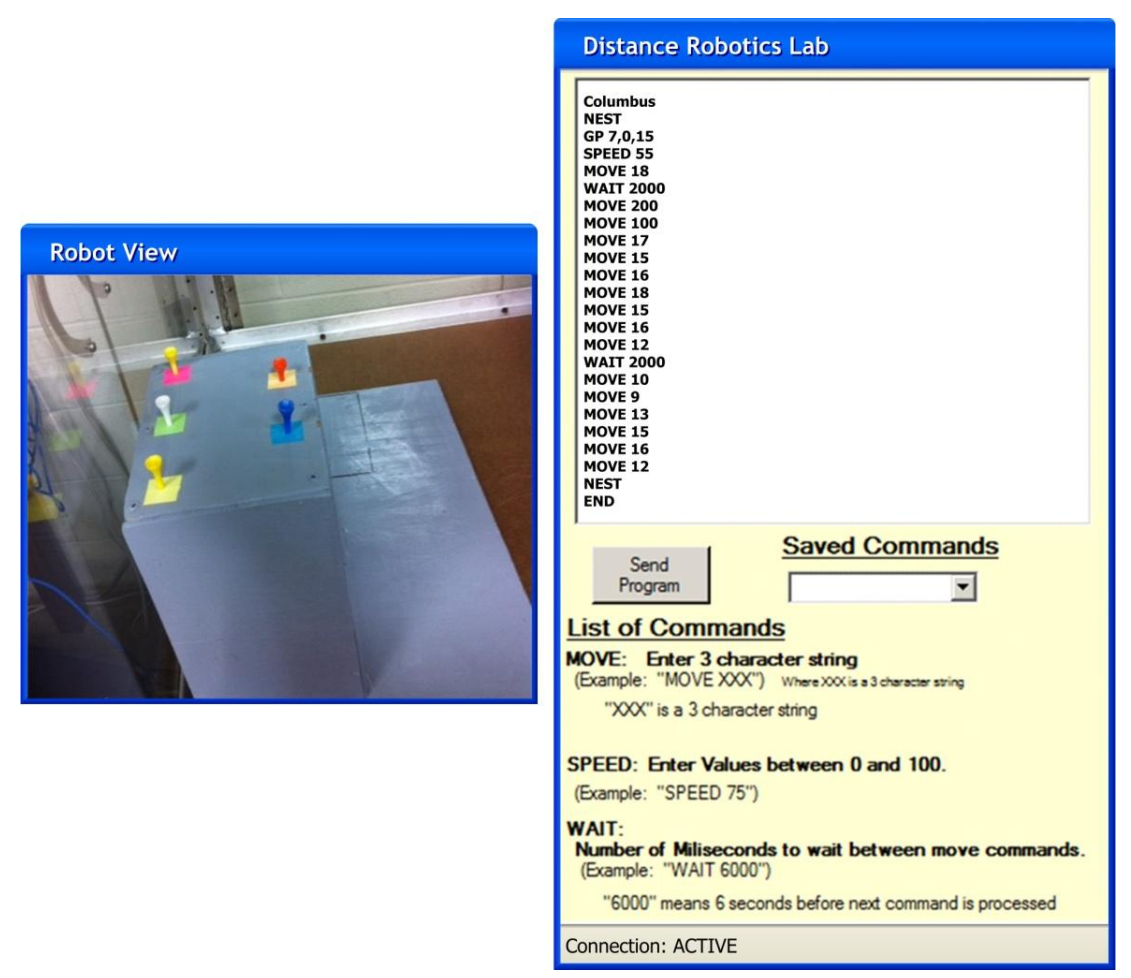

Figure 4: Client-side application interface in PROGRAMMING mode

\section{Robot Programming Laboratory Experiment}

The remote robotics laboratory system presented is mainly used for robot programming laboratory experiments in ENT 407 - Modern Manufacturing Systems course. The aim of these lab experiments is to demonstrate the operating practices actual industrial robot arm manipulators and introduce robot programming techniques to the engineering technology students. Upon completion of this lab, the students are expected to gain hands-on experience on programming and control of a full-scale articulated robot arm.

In order to perform the experiment, the students are first given the background information about the system operation. The students are informed that they will be using a robot arm located on the main campus remotely through their computers. They are required to download and install the remote-robotics client applications from the university website. They are given a short video lecture, video demonstration and instructions on how to operate the remote laboratory system software and the web-controlled camera. Once the students are familiarized with the system, they are provided instructions and tasks for completing the laboratory experiment.

For the beginning, the students are first expected to program the remote robot arm using the client-side control panel in order to remove five plastic pegs from a peg board and place them in a container. 


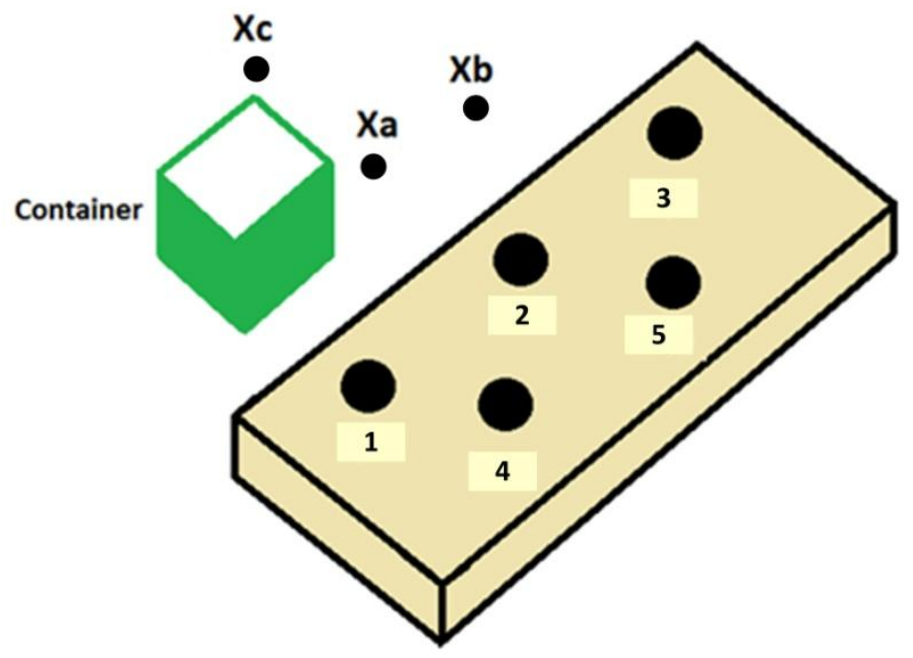

Figure 5: The possible locations for robot movements

A schematic diagram of the base and container is shown in Figure 5. The pegs are shown as the black holes on the base. The container is the green box shown on the left. The numbers next to the peg holes are used to identify the positions. There are three additional positions above the container and the base. Positions $\mathrm{Xa}$ is near the front of the box, $\mathrm{Xb}$ is behind it, and $\mathrm{Xc}$ is over the container.

A basic movement procedure for the robot to complete the given task should be as follows:

[1] Nest the robot arm (Move it to initial home position)

[2] Move the robot arm to $\mathrm{Xb}$ and then to \#1 for picking up Peg 1.

[3] Pick up the peg and move up to position $\mathrm{Xb}$.

[4] Move to position Xc through Xa

[5] Open the gripper to drop the peg in the container.

[6] Move back to Xb through Xa.

[7] Repeat steps 3 through 6 for the remaining four pegs.

Students first use the client-side manual control panel to save the robot positions needed for this motion sequences. As they move the robot using the screen controls, they observe the movements on the robot arm in real-time through the web-camera. Once the manual control part is completed, the students write a robot sequence program using basic robot commands (MOVE, SPEED, OPEN, CLOSE, DELAY) on the client-side control panel. The completed program is sent to the server computer for automatic execution and testing.

At the completion of the given robot tasks, the students must document their experiences with written formal lab reports. The lab report should briefly state and analyze the steps taken for completing the given tasks. The results of the experiment, i.e. recorded robot positions and completed robot programs should also be included. The students must also explain reflective statements in their report. They should state how effective was the client/server based remote access robotic lab station for them to get familiar and experienced with the industrial robotics and cellular manufacturing control. In addition to the lab reports, all the students who complete 
the lab with the remote robot laboratory system are asked to complete a qualitative questionnaire survey designed to capture students' response regarding their experience with the remote robotics laboratory.

\section{Assessment Study}

In order to measure the effectiveness of the presented remote robotics laboratory system in the distance education, a preliminary assessment study has been performed on the ENT 407 Modern Manufacturing Systems course. The ENT 407 is a 3-credit distance-learning course delivered in hybrid format. The course contents, lecture notes and assignment materials are provided to the students throughout the course web site. In-class lectures are delivered using the IVDL system and WebEx. The course covers a broad range of topics in manufacturing including the manufacturing processes, modern manufacturing technologies, application of computers in industry, non-manufacturing environments such as Group Technology, Lean Manufacturing, $\mathrm{CAD}$, and Concurrent Engineering. Due to the restrictions of its distance delivery format, no laboratory exercises had been previously developed for this course. The implementation of the remote robotics laboratory is the first practical implementation at this institution.

The assessment methodology combines use of multiple basic instruments including student laboratory reports, test scores and questionnaire surveys regarding the labs completed using the remote robotics laboratory system. The laboratory reports are submitted by each student individually by the week following the completion of the robot programming lab project.

The topics related to the robot programming are covered in the second midterm exam (Test 2) of the course. This test is given in the second half of the semester, typically after the completion of the robot programming lab. The assessment study presented here also involves the average scores for this test. Both the lab reports and tests are graded over 100 points. These grades are then converted to 0-4.0 scale for comparison purposes.

For experimentation, the performance of the students taking the ENT 407 course was analyzed in two groups: 1) Local Students, 2) Distance Students. All the course lectures, assignments, tests and laboratories are delivered equally to both student groups. The only difference between the two groups is that the local students are traditional on-campus students who are physically present on the main campus of the university. Local students can perform the lab using the physical equipment present in the lab. The distance students do not reside on the main campus. They can perform the robot programming lab experiment by using the remote robot station via the internet. They must use the developed client-side software application and operate the robot arm while watching its movement through the web camera. For completing the laboratory experiments, both groups of students are given equal amount of adequate time.

Tables 1 and 2 shows the summary results of the assessment performed with these two student groups. The presented analysis is based on the data gathered over three semesters from total of 65 students. It should be noted that the $20 \%$ of the total student population of this study consists of local students. 
Table 1: Summary of the Qualitative Questionnaire Survey Results

\begin{tabular}{|l|c|c|c|c|}
\hline \multirow{2}{*}{ RUBRICS } & \multicolumn{3}{|c|}{ GROUP 1: Local Students } & \multicolumn{2}{c|}{$\begin{array}{c}\text { GROUP 2: Distance } \\
\text { Students }\end{array}$} \\
\cline { 2 - 5 } & \multicolumn{2}{|c|}{ SD } & MEAN & SD \\
\cline { 2 - 5 } & MEAN & 0.54 & 3.5 & 0.58 \\
\hline $\begin{array}{l}\text { The lab sessions were motivating } \\
\text { for me to learn more about } \\
\text { Industrial Robotics systems. }\end{array}$ & 3.8 & 0.66 & 3.3 & $\mathbf{0 . 7 3}$ \\
\hline $\begin{array}{l}\text { The teaching method in lab } \\
\text { sessions helped me visualize and } \\
\text { understand the manufacturing } \\
\text { automation. }\end{array}$ & 3.6 & $\mathbf{0 . 9 8 7}$ & 3.2 & $\mathbf{0 . 9 6}$ \\
\hline $\begin{array}{l}\text { I got familiar with hands on } \\
\text { practice of industrial robots with } \\
\text { the lessons I learnt in this lab } \\
\text { experience. }\end{array}$ & 3.2 & $\mathbf{0 . 6 7 5}$ & 3.7 & $\mathbf{0 . 7 1}$ \\
\hline $\begin{array}{l}\text { This method helped me learn the } \\
\text { basics of industrial robot control } \\
\text { and programming. }\end{array}$ & $\mathbf{3 . 6}$ & $\mathbf{0 . 5 5}$ & 3.8 & $\mathbf{0 . 5 9}$ \\
\hline $\begin{array}{l}\text { I would like to have lab sessions } \\
\text { like this to help me learn. }\end{array}$ & $\mathbf{3 . 8}$ & $\mathbf{0 . 8 8}$ & $\mathbf{3 . 7 5}$ & $\mathbf{0 . 8 2}$ \\
\hline $\begin{array}{l}\text { Overall, I have had fun with this } \\
\text { lab. }\end{array}$ & $\mathbf{3 . 6}$ & & & \\
\hline
\end{tabular}

Table 2: Average Student Grades in ENT 407

\begin{tabular}{|c|c|c|}
\hline & $\begin{array}{c}\text { GROUP 1 } \\
\text { Local Students }\end{array}$ & $\begin{array}{c}\text { GROUP 2 } \\
\text { Distance Students }\end{array}$ \\
\hline $\begin{array}{c}\text { Grades for Robot Programming } \\
\text { Lab Report }\end{array}$ & $\mathbf{3 . 3 7}$ & $\mathbf{3 . 2 5}$ \\
\hline $\begin{array}{c}\text { Grades of Test 2 } \\
\text { (3.35 }\end{array}$ & $\mathbf{3 . 0 9}$ \\
\hline
\end{tabular}

An interesting conclusion can be drawn in relation to the comparative assessment between the two groups surveyed. The direct assessment results based on grades suggest that both student groups are getting similar learning experiences from the robot programming lab. There are no signicant performance differences between the two groups. Also, the relatively close assessment results of the test and laboratory reports are valid indicators that both groups grasp the subject matter. For the initial study, the results are found fairly satisfactory to prove that there are not significant differences in student responses to working with remote and local equipment. It is also recognizable from the qualitative survey results that the remote laboratory practice has somewhat a positive influence on the distance-learning students' motivation. 


\section{Conclusions}

An implementation and assessment of a remote robotics laboratory workstation system has been presented in this paper. The presented educational system has been utilized in an undergraduate engineering technology distance education course for providing laboratory training on industrial robotics.

Based-on the experience we gained with the presented system in distance-engineering technology-education, it is highly convenient and cost-effective to utilize remote laboratory technology in distance education. These systems enable delivering hands-on learning experience to a range of distance learning students, who do not have constant access to the university's laboratory facilities due to geographic restrictions.

A preliminary assessment study has been performed to investigate the effectiveness of the presented remote laboratory system in distance engineering technology education. This initial study uses standard quantitative and qualitative indicators to measure the effectiveness of the developed remote laboratory system in students' hands-on learning experience.

The presented assessment study compares the learning experiences gained by distance learning students using remote-access laboratory instruments with local students using physical instruments. The intention of this preliminary work is to validate the efforts for developing remote laboratory systems in order to provide laboratory training to the distance-learning students.

Overall; both qualitative and quantitative results of the preliminary assessment suggest that, the remote laboratory systems are highly effective in distance education as distance-learning students gain a similar experience to the local students. The remote laboratory system also contributes improvement of distance students' motivation.

It should be noted that, many other factors that influence students' learning outcomes are not measured in this preliminary study. A larger-scale study will be conducted in the future by the author in order to capture multiple influential factors and investigate the effectiveness of remote laboratories more profoundly.

\section{References}

1. Bal, M. Virtual manufacturing laboratory experiences for distance learning course in engineering technology. Paper session presented at the meeting of 2012 ASEE Annual Conference and Exposition, San Antonio, Texas, United States, June 2012.

2. Fujita, J. S. T., Cassaniga, R. F., And Fernandez, F. J. R. Remote laboratory. In Proceedings of the 2003 IEEE International Symposium on Industrial Electronics. Rio. de Janeiro, Brazil. 1104-1106, 2003.

3. Yoo, S. And Hovis, S. Technical symposium on computer science education. In Proceedings of the $35^{\text {th }}$ SIGCSE Technical Symposium on Computer Science Education. New York, NY. 311-314, 2004.

4. Scanlon, E., Morris, E., Di Paolo, T., And Cooper, M. Contemporary approaches to learning science: Technologically-mediated practical work. Studies in Sci. Education 38, 73-114, 2002.

5. Cooper M., Scanlon E. and Freake S. L. Remote controlled teaching experiments, in science and engineering subjects, accessible over the World-Wide-Web - The PEARL project, Proc. ED-MEDIA 2000 Conference, Montreal, Quebec, Canada, 2000. 
6. Ogot, M., Elliott, G., \& Glumac, N. An assessment of in-person and remotely operated laboratories. Journal of Engineering Education, 92(1), 57-62, 2003.

7. Sonnenwald, D. H., Whitton, M. C., \& Maglaughlin, K. L. Evaluating a scientific collaboratory: results of a controlled experiment. ACM Transactions on Human Computer Interaction, 10(2), 150-176, 2003.

8. Gomes, L., Coito, F., Costa, A., Palma, L., \& Almeida, P. Remote Laboratories support within Teaching and Learning Activities. International Conference on Remote Engineering and Virtual Instrumentation, 2007.

9. Accreditation Board for Engineering and Technology, http://www.abet.org.

10. Hergert D., Instrumentation Based Mobile Laboratories for an Electromechanical Engineering Technology Distance Education Program, Paper session presented at the meeting of 2010 ASEE Annual Conference and Exposition, June 20 - 23, 2010, Louisville, Kentucky, United States, 2010.

11. Mitsubishi Movemaster RV-M1 Technical Data and Manual: http://www.roboex.com/s19.html (Last Viewed: 3/13/2014).

12. Axis Network cameras website: http://www.axis.com/ (Last Viewed: 3/13/2014). 